\title{
Sacral Chordoma: Challenging for Resection Margin
}

\author{
Seung-Hyun Lee, Byung-Kwon Ahn \\ Department of Surgery, Kosin University College of Medicine, Busan, Korea
}

See Article on Page 122-131

A chordoma is a rare, bone neoplasm that accounts for $1 \%-4 \%$ of all bone neoplasms [1]. Although it considered to be a low-grade neoplasm with a slow-growing pattern, its clinical course is very aggressive, with a locally-invasive, highly-recurrent character [2]. It has been reported to be predominant in men, and its peak incidence is between 50-60 years of age [3]. It arises from the sacrococcygeal region in approximately $40 \%-50 \%$ of cases, from the base of the skull (spheno-occipital/nasal) in 35\%-40\% of cases, and from vertebral bodies in $15 \%-20 \%$ of cases $[2,4,5]$.

A chordoma usually present clinically with pain as the cardinal symptom. Neurologic symptoms tend to vary based on the location of the lesion. Jeys et al. [6] reviewed 33 patients with a chordoma arising from the sacrococcygeal region. Pain, typically dull and worse with sitting, was the most common presenting symptom in $85 \%$ of the patients. Pain commonly developed in the lower back and the sacrum. Other painful regions were the legs, buttocks, and the perianal and perineal regions. The classic symptoms of cauda equine (saddle anesthesia, leg parathesia, motor weakness, bladder dysfunction, bowel dysfunction) occurred in $70 \%$ of the patients. Urinary incontinence and outflow obstruction were frequent bladder dysfunction symptoms. The majority of bowel dysfunctions were due to constipation.

Unfortunately, these symptoms are nonspecific in nature, and a chordoma has a slow-growing pattern with an insidious onset of symptoms. Therefore, it is often found at the late stages of the disease. Jeys et al. [6] reported that the mean duration of symptoms prior to diagnosis was 120 weeks (2.3 years; range, $0.5-8.0$ years). The mean maximum tumor size at resection was $8.3 \mathrm{~cm}$, with a mean volume of $614 \mathrm{~cm}^{3}$.

Correspondence to: Byung-Kwon Ahn, M.D.

Department of Surgery, Kosin University College of Medicine,

262 Gamcheon-ro, Seo-gu, Busan 602-702, Korea

Tel: +82-51-990-6462, Fax: +82-51-246-6093,

E-mail:gsabk@hotmail.com

(C) 2014 The Korean Society of Coloproctology

This is an open-access article distributed under the terms of the Creative Commons Attribution NonCommercial License (http://creativecommons.org/licenses/by-nc/3.0) which permits unrestricted noncommercial use, distribution, and reproduction in any medium, provided the original work is properly cited.
Differential diagnosis is important for the surgical and the postsurgical management of a chordoma. The accurate diagnosis of tumors is of valuable prognostic significance. Fine-needle aspiration biopsy or core-needle biopsy in the case of bony lesions has been suggested to be the most oncologically sound approach to establish a diagnosis before resection, with care to avoid tumor seeding $[5,7]$. The excellent capabilities of magnetic resonance imaging and computed tomography allow precise delineation of tumors in terms of volume and anatomic characteristics, and aid in diagnosis [8]. In this study, three of eight patients were misdiagnosed and had undergone primary surgery with an R1 margin at other hospitals. After re-resection, all three cases had recurrences. On the contrary, four patients had been diagnosed with preoperative core-needle biopsy and underwent primary surgery with a R0 resection. One of the four patients had a local recurrence after 18 months. The patient had an initial $12.5-\mathrm{cm} \times 13.0$ $\mathrm{cm}$ tumor infiltrating the mesorectum [9].

With early accurate diagnosis, a complete en bloc resection with a $\mathrm{R} 0$ resection margin is a key point in the treatment of a chordoma. Rates of local recurrence, as well as survival, appear to depend on the achievement of a $\mathrm{R} 0$ resection margin. Tzortzidis et al. [10] reported that aggressive surgical approaches to achieve total resection resulted in long-term control in $50 \%$ of the cases. Aggressive surgical resection and reconstruction may require a multidisciplinary team approach.

\section{REFERENCES}

1. Healey JH, Lane JM. Chordoma: a critical review of diagnosis and treatment. Orthop Clin North Am 1989;20:417-26.

2. Bergh P, Kindblom LG, Gunterberg B, Remotti F, Ryd W, MeisKindblom JM. Prognostic factors in chordoma of the sacrum and mobile spine: a study of 39 patients. Cancer 2000;88:2122-34.

3. McMaster ML, Goldstein AM, Bromley CM, Ishibe N, Parry DM. Chordoma: incidence and survival patterns in the United States, 1973-1995. Cancer Causes Control 2001;12:1-11.

4. Fuchs B, Dickey ID, Yaszemski MJ, Inwards CY, Sim FH. Operative management of sacral chordoma. J Bone Joint Surg Am 2005; 87:2211-6.

5. Fourney DR, Gokaslan ZL. Current management of sacral chordoma. Neurosurg Focus 2003;15:E9. 
6. Jeys L, Gibbins R, Evans G, Grimer R. Sacral chordoma: a diagnosis not to be sat on? Int Orthop 2008;32:269-72.

7. Walcott BP, Nahed BV, Mohyeldin A, Coumans JV, Kahle KT, Ferreira MJ. Chordoma: current concepts, management, and future directions. Lancet Oncol 2012;13:e69-76.

8. Chen KW, Yang HL, Kandimalla Y, Liu JY, Wang GL. Review of current treatment of sacral chordoma. Orthop Surg 2009;1:238-44.
9. Garofalo F, Christoforidis D, di Summa PG, Gay B, Cherix S, Raffoul W, et al. The unresolved case of sacral chordoma: from misdiagnosis to challenging surgery and medical therapy resistance. Ann Coloproctol 2014;30:122-31.

10. Tzortzidis F, Elahi F, Wright D, Natarajan SK, Sekhar LN. Patient outcome at long-term follow-up after aggressive microsurgical resection of cranial base chordomas. Neurosurgery 2006;59:230-7. 